\title{
Instrumentos e integração de políticas públicas: a rede do Cadastro Único
}

\author{
Denise do Carmo Direito 12 \\ Natalia Massaco Koga 23 \\ 1 Universidade de Brasília, Brasília / DF - Brasil \\ 2 Ministério da Economia, Brasília / DF - Brasil \\ 3 Instituto de Pesquisa Econômica e Aplicada, Brasília / DF - Brasil
}

Este artigo analisa a capacidade de integração de políticas públicas suportadas por um mesmo instrumento de implementação. Partindo da abordagem dos estudos de implementação de políticas públicas que compreendem os instrumentos como "o estado em ação", isto é, ferramentas de ação pública por meio das quais a ação governamental se materializa e é operacionalizada (Lascoumes \& Le Galès, 2007; Linder \& Peters, 1990), examina-se o caso da Rede do Cadastro Único para Programas Sociais, formada pelo instrumento do Cadastro Único - composto por sua base de dados, normas, processos e estrutura municipal de cadastramento e coleta de dados - e pelos 20 programas sociais que se utilizam de suas informações cadastrais sobre 27 milhões de famílias, quase 80 milhões de pessoas de baixa renda, para seleção de beneficiários e gerenciamento de políticas voltadas ao combate das diferentes formas de vulnerabilidade social. Adotou-se a metodologia da análise de redes sociais para responder a duas perguntas principais: qual é a posição ocupada pelo Cadastro Único (CadÚnico) na rede de relações formada com as políticas sociais que o utilizam e quais são as condições e o nível de integração que o CadÚnico promove entre essas políticas usuárias? As métricas e os sociogramas de dois contextos temporais distintos, 2016 e 2018 , demonstraram que, embora haja potencial para que promova maior integração entre as políticas, o CadÚnico ainda não se apresenta como instrumento potente de integração de políticas públicas.

Palavras-chave: Cadastro Único para programas sociais; instrumento de política pública; integração de política pública; análise de redes sociais (ARS).

\section{Instrumentos e integración de políticas públicas: la red del Registro Único}

Este artículo analiza la capacidad de integración de políticas públicas sustentadas por un mismo instrumento de implementación. Partiendo del enfoque de los estudios de implementación de políticas públicas que comprenden instrumentos como "el estado en acción", es decir, herramientas de acción pública por medio de las cuales la acción gubernamental se materializa y se pone en marcha (Lascoumes \& Le Galès, 2007; Linder \& Peters, 1990), se examina el caso de la Red del Registro Único para Programas Sociales, formada por su base de datos, normas, procesos y estructura municipal de registro y recolección de datos y también por los 20 programas sociales que utilizan la información de registro de 27 millones de familias, casi 80 millones de personas de bajos ingresos, para selección de beneficiarios y gestión de políticas dirigidas al combate de las diferentes formas de vulnerabilidad social. Se adoptó la metodología de Análisis de Redes Sociales para responder a dos preguntas principales: 1) ¿Cuál es la posición que ocupa el Registro Único en la red de relaciones formada con las políticas sociales que lo utilizan? 2) ¿Cuáles son las posibilidades y el nivel de integración que el Registro Único promueve entre esas políticas? Las métricas y sociogramas de dos contextos temporales distintos -2016 y 2018- demostraron que, aunque haya potencial para que promueva una mayor integración entre las políticas, el Registro Único todavía no se presenta como instrumento potente de integración de políticas públicas.

Palabras clave: Registro Único para programas sociales; instrumento de política pública; integración de políticas públicas; análisis de redes sociales (ARS). 


\section{Instruments and public policy integration: the network of the Single Registry for Social Programs}

This article analyzes the capacity of policy integration through implementation tools. The research builds on studies on policy implementation, which understand such tools as "the state in action," i.e., instruments through which governments operate and deliver public services (Lascoumes \& Le Galès, 2007; Linder \& Peters, 1990). The research examines the case of the Brazilian Single Registry for Social Programs (Cadastro Único or CadUnico). The Single Registry is understood as part of a network providing social protection to the Brazilian population in social vulnerability. This work examines CadUnico as a complex system formed of a database and a set of norms and processes that work within the structure of local governments. The system gathers data used to coordinate 20 social programs, helping to select beneficiaries and manage policies that serve 27 million low-income families, reaching almost 80 million people. We adopted the social network analysis as a research method, seeking to answer two main questions: what is the position occupied by the Single Registry in the network of relationships formed by the federal social policies which use it, and what are the conditions and the level of integration which it promotes among these federal user policies? The metrics and sociograms of two distinct timeframes, 2016 and 2018, have demonstrated that, although the Single Register for Social Programs offers elements to promote some integration among public policies, there is no evidence of its effectiveness to this end.

Keywords: Single Registry for Social Programs; policy tool; policy integration; social network analysis (SNA).

\section{INTRODUÇÃO: UM INSTRUMENTO, MÚLTIPLOS PROGRAMAS}

O Cadastro Único para Programas Sociais (CadÚnico) foi criado pelo Decreto nº 6.135, de 25 de junho de 2007, com dois objetivos principais: identificação e caracterização socioeconômica das famílias brasileiras de baixa renda brasileiras e integração de programas sociais do Governo Federal voltados ao atendimento da população de baixa renda. ${ }^{1}$ Em relação à primeira finalidade, pode-se afirmar que o CadÚnico desempenhou papel fundamental na rápida expansão do Programa Bolsa Família (PBF) e que, no decorrer dos anos, foi paulatinamente sendo adotado como fonte de dados para a seleção de beneficiários de diversas políticas sociais. No entanto, pouco se conhece sobre o segundo papel: a capacidade de integração desse instrumento que alimenta diversas políticas públicas.

O CadÚnico coleta e disponibiliza informações atualizadas periodicamente para 20 programas sociais federais a respeito de um amplo quadro de informações socioeconômicas de 27 milhões de famílias, quase 80 milhões de pessoas de baixa renda, isso é, famílias com rendimento menor ou igual a meio salário mínimo.

É recente e ainda escassa a produção de estudos que examinem o CadÚnico como um objeto de análise próprio, uma vez que no início de sua existência era considerado, dentro das pesquisas sobre o PBF, um suporte de implementação do programa no papel especificamente de instrumento de seleção de beneficiários (Direito, Koga, Lício, \& Chaves, 2016, 2018). Contudo, ao longo dos mais de 10 anos, o CadÚnico passou a servir e interagir com dezenas de programas federais e com um número ainda desconhecido de programas estaduais e municipais. Este estudo busca descrever e explorar esse novo contexto interacional do CadÚnico, analisando seu papel como instrumento de integração de políticas públicas, previsto em sua criação, mas ainda não explorado em estudos empíricos.

\footnotetext{
${ }^{1}$ Os artigos $2^{\circ} \mathrm{e} 3^{\circ}$ do Decreto no 6.135 tratam do cadastro como meio de fazer a integração entre programas e políticas públicas que, por isso, devem utilizá-lo de forma obrigatória para a seleção debeneficiários. Além disso, outrasiniciativas, como ainstituição da Rede Cadastro Uninico, em novembro de 2017, pretendia "melhorar a coordenação das políticas sociais do governo federal", conforme matéria da Agência Brasil recuperado de http://www.brasil.gov.br/cidadania-e-justica/2017/05/cidadaos-tem-acesso- a-dados-do-cadastro-unico-na-internet?TSPD_101_ $\mathrm{R} 0=54 \mathrm{c} 0 \mathrm{c} 9 \mathrm{e} 659 \mathrm{~d} 528 \mathrm{~d} 85 \mathrm{a} 6868363 \mathrm{~d} 16 \mathrm{~b} 1 \mathrm{bcyA} 800000000000000006 \mathrm{fb} 3 \mathrm{~b} 0 \mathrm{bfffff} 00000000000000000000000000005 \mathrm{~d} 2 \mathrm{e} 3$ 3590058322c9208282a9212ab2000a8273c9c90c82c5b32318a8c6a66fb1f66c12533afc86fc42efc6eb876a7dda208d40c58ec0a2800be2aeab 10c374d9e27f428ec1ac34eef436a021121544f46b369f9775f7e72e3b7364118305d20b0dessa
} 
Como instrumento de integração de políticas, o CadÚnico poderia gerar diversos efeitos no processo de implementação de políticas, como redução do custo de implementação, melhora de focalização, ofertas mais adequadas de políticas sociais, além de facilitar o acesso ao cidadão aos benefícios sociais (Barca \& Chichir, 2014; Direito et al., 2016). Pretende-se nesta pesquisa, portanto, responder a duas principais perguntas: qual é a posição ocupada pelo CadÚnico na rede de relações formada com as políticas sociais federais que o utilizam e quais são as condições e o nível de integração que o promove entre essas políticas usuárias federais?

Como detalham Direito et al. (2018), o instrumento do CadÚnico é composto por um conjunto de mecanismos de implementação, como normas, postos de atendimento municipais, formulários de coleta de dados e sistema informatizado. ${ }^{2}$ A presente análise considera o CadÚnico o conjunto desses mecanismos, e não apenas um simples banco de dados. Argumenta-se que a vinculação do cadastro com os programas usuários não se configura só por meio da base de dados. Em verdade, os outros mecanismos acima mencionados também condicionam a implementação dos programas usuários. Por exemplo, as normas de funcionamento do cadastro ${ }^{3}$ estabeleceram condições, estruturas e processos de funcionamento que afetam a implementação e o nível de integração dos programas usuários, motivo pelo qual são tratadas nesta pesquisa como parte do instrumento do CadÚnico.

\section{REVISÃO DA LITERATURA}

A revisão da literatura explora dois conjuntos de abordagens teóricas que ajudam a compreender a natureza do CadÚnico e a complexidade para a sua adequada análise. O primeiro discorre sobre a literatura de instrumentos de políticas públicas, justificando o enquadramento do CadÚnico como um instrumento de ação pública e a relevância da análise de sua potencialidade como instrumento de integração de políticas públicas. O segundo traz elementos da abordagem relacional da implementação de políticas para o exame dessa potencialidade transacional do CadÚnico.

\subsection{Instrumentos de Políticas Públicas}

Os instrumentos representam diferentes níveis de intervenção estatal e são materializados de formas distintas: normas, regulamentos, incentivos, especificações técnicas, ferramentas, entre outros (Lascoumes \& Le Galès, 2007). Como visto, o cadastro apresenta vários desses níveis de intervenção. De forma resumida, podemos dizer que ele apresenta normas, ${ }^{4}$ atua numa realidade interfederativa que conta com desenvolvimento de capacidades para o correto preenchimento dos dados e o desempenho das ações de cadastramento, bem como conta com um sistema informacional que dá suporte às tarefas e materialidade à política.

As várias definições de instrumentos de implementação têm em comum o fato de identificaremnos como "ação governamental". Tais instrumentos, portanto, indicam como o estado interfere na

\footnotetext{
${ }^{2}$ Para mais informações sobre cada uma dessas dimensões do CadÚnico, veja Direito et al. (2018).

${ }^{3}$ Para além do Decreto no 6.135/07, que instituiu o CadÚnico, há um conjunto de normas complementares, como a Portaria no 501, de 29 de novembro de 2017, que estabelece uma séria de condições e procedimentos que devem ser seguidos para que os programas usem dados e condicionem, em alguma medida, a forma de implementação deles.

${ }^{4}$ Principais normas: Decreto $n^{\circ} 6.135$, de 26 de junho de 2007 , e as portarias $n^{\circ} 376$, de 16 de outubro de 2008 , e $n^{\circ} 177$, de 16 de junho de 2011, além de instruções operacionais. Recuperado de http://mds.gov.br/assuntos/cadastro-unico/legislacao\#b_start=0
} 
sociedade e com quais objetivos. Dessa forma, analisá-los é compreender como o governo faz com que os atores sociais ajam da maneira desejada, visto que instrumentos de políticas públicas são iniciativas governamentais que buscam organizar/normatizar/incentivar ou coibir determinados comportamentos sociais.

As análises sobre instrumentos estiveram relegadas a uma área secundária dos estudos de políticas públicas, como parte dos exames de implementação de políticas públicas (Sabatier, 1986). Isso se alterou ao longo dos anos, principalmente a partir de abordagens feitas nos Estudos Unidos (King, 2007; Peters \& Van Nispen, 1998; Salamon, 2002), no Canadá (Howlett, 2000, 2009), na Inglaterra (Hood, 1983 e 2007) e, mais recentemente, na França (Lascoumes \& Le Galès, 2005, 2007).

Diferentes ensinamentos foram sendo criados com base nessas análises. O primeiro, denominado por Brujin e Hufen (1998) de "clássico", voltava-se inicialmente para a mera identificação e tipificação dos instrumentos. Como esclarece Chistopher Hood $(1983,2007)$, pioneiro nesse primeiro conjunto de estudos, tal abordagem se caracteriza pelo esforço de vários teóricos para simplificar e agregar instrumentos de forma a possibilitar a compreensão e a similaridade da adoção de determinadas ferramentas para a solução de questões de políticas públicas.

Ao longo das décadas, novas frentes de análise foram se formando, como a abordagem de instrumento-contexto, que considera as características contextuais que limitam ou propiciam a escolha do instrumento (Brujin \& Hufen, 1998). Soma-se a essas perspectivas a abordagem politics-of-instrumentality, que adiciona a dimensão política nas análises, sendo o instrumento um dos fatores que determinam o curso da política. $\mathrm{O}$ foco se amplia de tal forma que é necessário compreender o sistema de políticas, considerando variáveis como em que rede está inserido, as arenas de disputas, o processo de implementação, e, de igual forma, compreender as razões ideológicas ou de formato de política que levaram à adoção de determinado instrumento.

Linder e Peters (1990) defendem uma quarta abordagem, que denominaram de "construtivista", na qual aspectos menos evidentes para o uso de determinados instrumentos devem ser analisados. Assim, questões simbólicas, étnicas e, de igual forma, valores e percepções devem ser considerados quanto ao impacto do uso de certo instrumento. Dessa forma, não há uma realidade objetiva nem uma escolha racional, mas conhecimento imperfeito da realidade, e a legitimidade do instrumento será adquirida no tempo, durante sua operação (Linder \& Peters, 1990).

A ampliação do escopo analítico ao longo do tempo deixa claro que a definição e o uso de um instrumento não são um exercício mecânico em que se "encaixam" um problema e uma solução bem-definidos. Trata-se de um processo de construção de compreensão da realidade e da tentativa de trabalhar essa realidade. Lascoumes e Le Galès (2005) reforçam a dimensão cognitiva dos instrumentos, no sentido de que a escolha e o uso do instrumento demostram como o Estado quer intervir na sociedade.

Neste artigo, adotaremos a abordagem sociológica desenvolvida por Lascoumes e Le Galès, que compreendem os instrumentos como ferramentas de ação pública por meio dos quais a ação governamental se materializa e é operacionalizada (2007). Dentro dessa perspectiva, a ação pública se desenvolve num espaço sociopolítico, com finalidades e objetivos - uma abordagem mais funcionalista -, mas também por projetos de autor, ou seja, por opções possíveis adotadas pelos atores envolvidos no momento da definição, considerando-se seus valores e suas preferências, bem como os impactos gerados sobre a sociedade sujeita da ação.

Assim, para além de compreender os motivos que determinaram a escolha do instrumento, é tão ou mais relevante entender as consequências que tais escolhas acarretaram na ação pública e nos 
próprios cidadãos. Para Lascoumes e Le Galès (2005), “a instrumentação da ação pública é, portanto, um meio de orientar as relações entre a sociedade política (via executivo-administrativo) e a sociedade civil (via sujeitos administrados) por intermediários, dispositivos misturando componentes técnicos (medida, cálculo, regra de direito, procedimentos) e sociais (representação, símbolo)".

A análise dos instrumentos de implementação é uma forma de "desvendar" a ação pública, pois revela a compreensão do governo sobre aquela questão, por isso a mudança do instrumento significa modificação nos objetivos ou nos resultados que se pretendia alcançar. Os instrumentos não são neutros ou adotados sob uma tecnicidade asséptica, e sim escolhidos com base numa lógica específica, portanto apresentam efeitos e consequências.

No caso específico do CadÚnico, buscamos analisar sua concepção e potencialidade como instrumento de integração de políticas públicas. Como esclarece Guy Peters (2015), integração de políticas públicas seria um nível mais avançado de coordenação de políticas públicas em que cada decisão leva em consideração seus efeitos em todo o conjunto de organizações e programas a ela associado, assim como a consistência dessa escolha aos resultados comuns que se pretende alcançar. Em outras palavras, um nível de integração das políticas públicas demandaria coerência nos instrumentos e nos objetivos (Dobuzinskis \& Howlett, 2018; Peters, 2015). Tal coerência deveria ser refletida, assim, ex-ante, desde a formulação dos programas e dos instrumentos e acompanhada ao longo do processo de implementação, garantindo que todas as ações estejam voltadas para a mesma direção e que haja correções de percursos caso a interação entre as diversas ações esteja gerando efeitos contraditórios.

Em que pese a maior dificuldade para alcançar tal nível de coordenação, segundo Peters (2015), problemas públicos abrangentes - como a própria inclusão social, finalidade atacada pela rede do CadÚnico - poderiam funcionar como elemento facilitador para a mobilização e a condução das organizações e suas ações. Contudo, como o autor esclarece, a questão base da coordenação estaria no equilíbrio entre especialização e segmentação do governo. Ou seja, qual seria o limite da especialização das políticas públicas que ainda permita enxergar o whole citizen, o cidadão como um todo, e não de forma segmentada, considerando apenas uma ou algumas de suas facetas? O CadÚnico aparenta ser um instrumento a lidar com esse desafio, uma vez que teria como finalidade específica trazer um olhar holístico das vulnerabilidades sociais.

Para compreender o CadÚnico como instrumento integrador de várias políticas, é imprescindível conhecer seu contexto transacional. Esse argumento será desenvolvido nas duas subseções a seguir, que justificarão a abordagem relacional e o método de análise de redes sociais para esta pesquisa.

\subsection{A Abordagem Relacional nos Estudos de Implementação de Políticas Públicas}

Cada vez mais, os estudos de implementação vêm explorando abordagens analíticas menos racionais e lineares, passando a incorporar novas dimensões explicativas ao fenômeno da política pública, como o aspecto relacional. Emirbayer (1997), um dos primeiros defensores da perspectiva relacional ${ }^{5}$

\footnotetext{
${ }^{5}$ Esta abordagem foi se formando por uma série de trabalhos empíricos que focaram no aspecto transacional e na dinâmica dos fluxos entre entes sociais, realizados na Europa e nos Estados Unidos. Ao longo das últimas três ou quatro décadas, esses estudos foram ganhando maior densidade e puderam se consolidar numa perspectiva analítica com pressupostos próprios. A despeito das diferentes terminologias adotadas ao longo dessa trajetória - como relacionalismo em contraposição a substancialismo (Emirbayer, 1997), análise estrutural chamando a atenção para a forma e o conteúdo das estruturas relacionais (Wasserman \& Faust, 1994; Wellman, 1997) ou, ainda, análise de redes sociais que enfatizam o poder do método de análise (Knoke \& Yang, 2008) -, este trabalho utilizará a expressão "abordagem relacional" para fazer referência ao conjunto de princípios e instrumentos analíticos que envolvem esse campo de exploração da dimensão transacional entre os entes sociais.
} 
para a análise da realidade social, apontou que a abordagem relacional se contrapõe à concepção substancialista - adotada por correntes como as teorias da escolha racional e da conformidade normativa -, que entende os entes sociais como estáveis, pré-formados e constituídos por atributos inerentes e imutáveis. A abordagem relacional, em contraste, defende que os entes sociais são, na realidade, um produto das relações nas quais estão imersos e, portanto, não podem ser analisados separadamente de seus contextos transacionais (Emirbayer, 1997). Dessa forma, a abordagem relacional redefine a unidade de análise de uma pesquisa que deixa de ser o indivíduo, suas estratégias ou normas para se voltar às relações sociais. Ou seja, o ponto de partida da análise da ação social na abordagem relacional é o contexto transacional nos quais os entes sociais estão imersos.

As bases da abordagem relacional podem ser sintetizadas em três grandes argumentos, como descrevem Knoke e Yang (2008). O primeiro deles é a compreensão de que, em geral, as relações estruturais sociais são recursos mais poderosos de explicação da ação social do que de atributos individuais, uma vez que percebe nuances e mudanças em contextos relacionais diversos que não são perceptíveis na observação de características específicas. O segundo pressuposto consiste no entendimento de que redes sociais afetam percepções, crenças e ações por meio de uma variedade de mecanismos estruturais constituídos por relações sociais. Contatos diretos e interações mais intensivas, por exemplo, colocam entes em melhores posições informacionais e de influência, enquanto contatos indiretos por meio de intermediários podem expor entes a novas ideias e recursos. Por fim, o terceiro pressuposto é o de que relações sociais devem ser vistas como processos dinâmicos, isto é, a ação dos entes sociais também transforma as estruturas relacionais que estão, assim, em constante transformação.

A abordagem relacional reconhece que as trocas entre entes sociais afetam o fluxo de bens materiais, ideias, informações e poder, determinando, portanto, a constituição das situações sociais. O universo do campo relacional e a posição dos indivíduos, assim, não são determinados aleatoriamente. $\mathrm{Na}$ verdade, eles são definidos pelas possibilidades e pelos limites fornecidos por trajetórias prévias. Dessa forma, "as relações e as posições nas redes constituem estruturas relacionais que constrangem escolhas, dão acesso diferenciado a bens e instrumentos de poder, tornam certas alianças ou conflitos mais ou menos prováveis e influenciam os resultados da política” (Marques, 2006).

\subsection{Instrumento do Cadastro Único: Um Sistema Complexo}

A abordagem relacional pode agregar vantagens para a análise do objeto específico deste trabalho, o CadÚnico, especialmente se esse for observado como um sistema complexo no sentido analisado por Furtado, Sakowski, e Tóvolli (2015). Para o autor, um sistema complexo tem três características. Primeira, a soma das partes que formam esse sistema não pode ser descrita pela análise de cada parte isolada. Assim, o "todo" não só é mais, como é diferente da soma das partes (Anderson, 1972 como citado em Furtado et al., 2015). Analisar parte por parte das políticas que compõem o sistema do CadÚnico não nos dará a visão do funcionamento do todo.

A segunda característica é que o sistema não tem necessidade de controle central e pode se autoorganizar; há uma sincronização e uma coordenação da política com base nos instrumentos e na forma como a política é implementada. Apesar de o CadÚnico ser um nó central dessa rede, visto que é dele que sai uma enorme gama de informações para a atuação das várias políticas, há arranjos entre os autores que formam novas redes e caminhos alternativos, constituindo esse ecossistema. Assim, não é adequado dizer que há um "controle" central. 
A terceira característica de sistemas complexos é que eles se transformam no tempo, ou seja, se adequam às possibilidades/impossibilidades no decorrer dos anos. Assim, são adaptáveis e evolutivos (Furtado et al., 2015).

Uma metodologia para conseguir abarcar o desafio de analisar um sistema com essas características necessita compreender o CadÚnico para além de suas estruturas formais. É necessário explicitar a forma como se relacionam os atores e as instituições que negociam e especificam o funcionamento de cada parte, bem como retratar a dinâmica do sistema, que é mais do que a simples soma das partes.

Dessa forma, para compreender o papel exercido pelo CadÚnico, torna-se imprescindível conhecer seu contexto transacional - sua composição, seus fluxos e suas dinâmicas. A abordagem relacional tem como vantagem para este estudo o fato de estar situada num plano de análise intermediário que permite a observação simultânea tanto das ações quanto das estruturas sociais (Marques, 2006). Ou seja, possibilita que sejam considerados na rede de transações do CadÚnico não só seus usos imediatos pelos diversos programas sociais, mas também a influência das estruturas desse conjunto de programas e a do próprio CadÚnico, assim como da ação conjunta de todos esses entes no produto interacional.

Com base nessas múltiplas dimensões e em consonância com a concepção sociológica de instrumentos proposta por Lascoumes e Le Galès $(2005,2007)$ é que analisamos o instrumento do CadÚnico como um sistema complexo em permanente interação, no sentido da influência da ação nas políticas, da ação das políticas no cadastro e da interação entre as próprias políticas que dele se alimentam.

Para compreender o papel integrador do instrumento do CadÚnico, esta pesquisa explorará seu contexto transacional, utilizando o método de análise de redes sociais para traçar os fluxos de informação e dinâmicas de acesso aos dados e a forma como esses dados chegam até o cidadão, a fim de que este possa ser beneficiário das políticas.

\section{METODOLOGIA}

O método utilizado neste exame foi a análise de redes sociais (ARS), que busca padrões de interação entre entes sociais (Freeman, 2002; Scott, 1992) e é comumente aplicada a estudos que partem da abordagem relacional acima descrita.

A ARS permite a reprodução gráfica e a análise de redes de relação entre atores e a mensuração de distintas características relacionais, como papéis e centralidade dos atores, assim como formação de subgrupos na estrutura relacional. As definições das métricas usadas para explorar tais características serão detalhadas na seção seguinte, ao se descreverem os resultados da exploração de cada uma delas.

A coleta dos dados foi feita por meio de aplicação de questionário sociométrico semiestruturado, aplicado em 29 entrevistas realizadas com gestores das políticas usuárias do CadÚnico, em março de 2016 e junho de 2018. Em 2016, obtiveram-se dados de 17 programas usuários e, em 2018, de 20. O mesmo questionário foi aplicado nos dois períodos de coleta. ${ }^{6}$

\footnotetext{
${ }^{6}$ Os seguinte programas foram considerados nas pesquisas de 2016 e 2018: Assistência Técnica Rural; Carteira do Idoso; Crédito da Reforma Agrária; Facultativo de Baixa Renda; Fomento às Atividades Rurais; ID Jovem; isenção de taxa de concurso público; Minha Casa, Minha Vida; Programa Bolsa Família (PBF); Programa Cisternas; Programa de Erradicação do Trabalho Infantil; Programa Nacional de Crédito Fundiário; Reforma Agrária; Serviço de Convivência e Fortalecimento de Vínculos; Tarifa Social de Energia Elétrica; Telefone Popular; TV digital. Programas que não existiam ou não usavam o CadÚnico em 2016 e por isso não foram considerados no respectivo sociograma: ação de distribuição de cesta; benefício de prestação continuada; isenção de taxa de inscrição para o Exame Nacional do Ensino Médio (Enem); Plano Progredir; Programa Criança Feliz. Programa descontinuado e que, por isso, não consta no estudo de 2018: Bolsa Verde.
} 
Foi solicitado aos entrevistados que informassem de quem recebiam bases/dados/informações e para quem os remetiam, até que se chegasse ao "último" usuário, que seria o órgão responsável pelo atendimento final ou pela concessão do benefício. A fronteira da rede construída nesta pesquisa foram os órgãos/instituições que fazem a entrega ao cidadão. ${ }^{7}$

As informações sobre "de quem recebo as informações e para quem as passo" foram reproduzidas numa matriz binária, em que foi identificado como um 1 os casos nos quais há envio de informações e 0 os casos sem envio de informações. A matriz foi analisada por meio da aplicação de softwares de análise de redes (Ucinet e Gelphi) e de visualização de redes (Netdraw). Com esses softwares foi possível gerar sociogramas que reproduzem os fluxos de troca de dados e informações estabelecidos entre os órgãos gestores dos programas federais usuários do CadÚnico e as fontes de dados utilizadas para a gestão dos programas - o próprio CadÚnico, outro registro administrativo ou, ainda, algum sistema de informações específico.

A coleta de dados permitiu mapear o fluxo de informações estabelecido entre o Cadastro e os programas usuários para a concessão de benefícios sociais aos cidadãos de baixa renda em dois momentos distintos: 2016 e 2018. O caráter longitudinal do estudo permitiu a comparação entre as redes formadas em 2016 e 2018, para a análise da estabilidade da estrutura relacional do CadÚnico e para a identificação de mudanças na capacidade do CadÚnico de promover integração das políticas públicas usuárias. Ademais, a comparação entre a rede de 2016 e a de 2018 possibilitou confrontar uma crítica usualmente feita ao método da ARS quanto ao privilégio da espacialidade em relação à temporalidade, uma vez que o método permitiria apenas fotografias das estruturas relacionais. Nesse caso, a comparação entre as duas fotografias permitiu avançar em relação à mera análise descritiva de uma estrutura relacional para gerar análises de modificação estrutural.

\section{RESULTADOS}

A comparação inicial das redes de 2016 e 2018 demonstra que houve um crescimento de quase $25 \%$ no número total de programas que utilizavam de forma sistemática o CadÚnico como instrumento de implementação, com a entrada de 5 novos programas na rede - destes, 2 já existiam, mas não usavam o CadÚnico, e os outros 3 foram lançados no período.

Em relação ao número de nós, ou seja, atores envolvidos no tratamento de informações e implementação da política, o crescimento foi um pouco menor: aproximadamente $20 \%$ - de 62 , em 2016, para 74, em 2018. De igual forma, o número de conexões (laços) também apresentou crescimento de $20 \%$ - de 145, em 2016, para 174, em 2018. Assim, houve um aumento ligeiramente superior no número de políticas em relação ao de atores, o que sugere a simplificação de fluxos - por exemplo, com transmissão direta das informações do cadastro para um programa que faça a entrega ao cidadão - ou a otimização e o uso de estruturas já existentes, em vez da criação de novas, o que evidenciaria maior integração das políticas públicas.

\footnotetext{
${ }^{7}$ Esse procedimento lida com uma das críticas ao método de ARS quanto às definições das fronteiras da estrutura relacional. Por serem fluidas e dinâmicas, as fronteiras das redes sociais são abstrações necessárias que, em alguma medida, reduzem a reprodução do contexto interacional. Neste estudo, a delimitação da fronteira da rede analisada até o limite da entrega do benefício ao cidadão se justifica pelo objeto do estudo que pretende avaliar a capacidade do CadÚnico de integrar políticas públicas.
} 
Medidas da rede e dos nós serão analisadas a seguir para aclarar o nível de integração entre o cadastro e os programas sociais federais.

\subsection{Medidas da Rede}

A rede do CadÚnico é composta por nós (atores), ligações e fluxos de informação, em que o direcionamento das ligações entre os atores importa, indicando quem manda informações, quem só recebe e quem manda e recebe, podendo limitar ou facilitar caminhos possíveis para acessar determinados atores. Dessa forma, medidas como diâmetro e distância geodésica são influenciadas por essa característica.

\section{TABELA 1 MEDIDAS DA REDE}

$\begin{array}{ccc} & \text { Rede } 2016 & \text { Rede } 2018 \\ \text { Média geodésica } & 4 & 3,4 \\ \text { Densidade } & 0,038 & 0,032 \\ \text { Diâmetro } & 10 & 8\end{array}$

Fonte: Elaborada pelas autoras.

Como mencionado, os dados demonstram que houve crescimento no número de programas entre os dois períodos. Contudo, percebe-se na Tabela 1 uma pequena redução na densidade. Essa medida retrata o número de ligações diretas em relação ao total de ligações possíveis. Considerando que redes definidas como densas são aquelas em que há grande quantidade de conexões e que redes esparsas são aquelas com pequenas quantidades de ligações, quanto maior a densidade, maior a coesão entre o grupo (Hatala, 2006).

Com o crescimento da rede, a redução na densidade só não ocorreria se houvesse um aumento significativo de ligações diretas, um aumento na interconexão dos atores, o que não ocorreu.

Chama a atenção também a redução do diâmetro. Essa medida retrata o número total de passos entre um extremo e outro da rede. Na terminologia usual de ARS, trata-se da geodésica - distância entre um nó e outro - mais longa naquela rede, considerando o caminho mais curto. Assim, o diâmetro de um grafo é a maior distância geodésica entre todos os pares de nós presentes (Silva, Matheus, Parreiras, \& Parreiras, 2006).

Apesar de a rede ter "crescido", a distância entre uma ponta e outra se reduziu, o que pode significar a construção de outras ligações que acarretaram a diminuição do diâmetro. A geodésica é a medida da distância entre os atores da rede. Essa medida mostra, em média, por quantos atores a informação precisa passar até chegar ao destino final. 


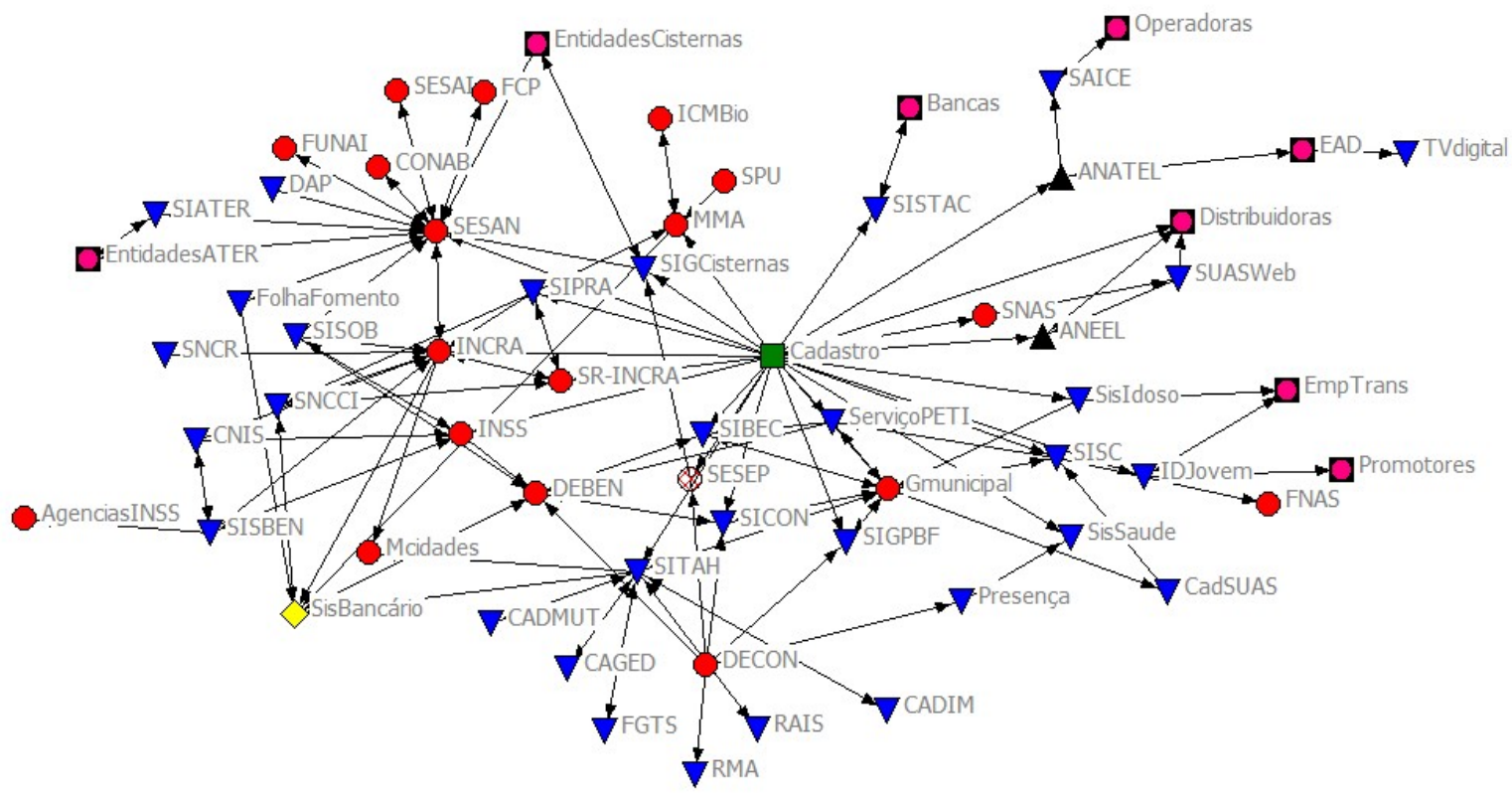

Fonte: Elaborada pelas autoras.

FIGURA 2 SOCIOGRAMA DA REDE DO CADASTRO ÚNICO EM 2018

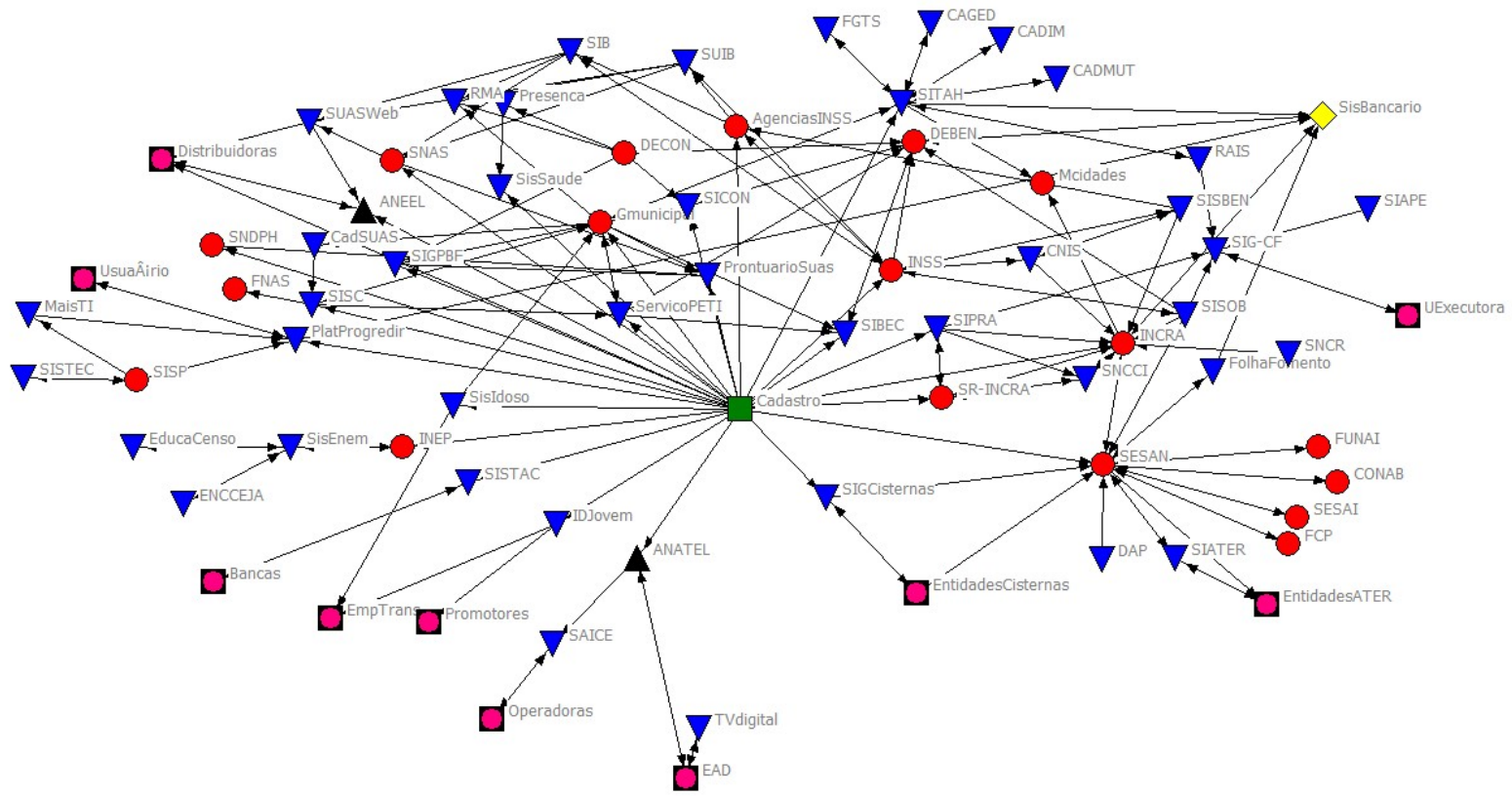

Fonte: Elaborada pelas autoras. 
A observação dos sociogramas demonstra que a maior parte dos contatos que o cidadão tem com os fornecedores das políticas sociais é, na verdade, com entes não governamentais, como bancas de concurso público, empresas de transportes, operadores da arte, bancos etc. As duas exceções são a gestão municipal, que faz a entrega dos serviços socioassistenciais, e as agências do INSS, que operam o BPC.

Como dito, uma ferramenta com as características do CadÚnico não pode ser considerada isenta de significados. Para além da redução de custos - um mesmo esforço de cadastramento para usuários dos programas -, deve ser considerada a "distância/impessoalidade" que a gestão central dos programas passa a ter em relação ao público beneficiário, com uma perda relativa de poder dos "donos" dos programas, que possivelmente é compensada por uma gestão mais efetiva e menos onerosa da política.

\subsection{Medidas dos Nós}

Nesta seção, serão analisadas as medidas típicas dos nós (atores). Em função da similaridade dos sociogramas, serão analisadas as medidas da rede do CadÚnico de 2018. Caso haja alguma característica distinta na rede de 2016, esta será apontada.

O ator é central numa rede quando pode se comunicar diretamente com muitos outros, quando está próximo de muitos atores ou, ainda, quando há muitos atores que o utilizam como intermediário em suas comunicações. Atores que têm mais ligações que outros podem estar em posição mais vantajosa. Por terem muitas ligações, apresentam formas alternativas para satisfazer às necessidades e aproveitar os recursos da rede; assim, têm menor dependência em relação a outros (Tomaél \& Marteleto, 2006).

As métricas de centralidade de grau de entrada (indegree) e de saída (outdegree) são medidas que retratam a quantidade de conexões recebidas ou geradas por um nó. Dessa forma, em grafos direcionados, mais central será o nó quanto maior for o número de conexões recebidas (no caso do indegree) ou geradas (no caso do outdegree).

\section{TABELA 2 REDE 2018 (OUTDEGREE)}

$\begin{array}{cc}\text { Cadastro } & 26 \\ \text { Gmunicipal } & 9 \\ \text { Sitah } & 8 \\ \text { Sesan } & 8 \\ \text { INSS } & 7 \\ \text { Incra } & 5 \\ \text { Sisob } & 5 \\ \text { Decon } & 5\end{array}$

Fonte: Elaborada pelas autoras. 


\section{TABELA 3 REDE 2018 (INDEGREE)}

$\begin{array}{cc}\text { Sesan } & 12 \\ \text { Incra } & 10 \\ \text { Deben } & 7 \\ \text { Sitah } & 7 \\ \text { INSS } & 6 \\ \text { Gmunicipal } & 6 \\ \text { SIG-CF } & 5 \\ \text { Sis. Bancário } & 5\end{array}$

Fonte: Elaborada pelas autoras.

Reproduziram-se, nas Tabelas 2 e 3, os valores dos 8 principais atores de cada uma das medidas. É necessário ressaltar alguns aspectos. Primeiro, a importância de alguns atores para a articulação de determinadas áreas, visto que aparecem tanto como fornecedores de dados quanto como receptores de informação, em especial, os nós Secretaria Nacional de Segurança Alimentar e Nutricional (Sesan), Instituto Nacional de Colonização e Reforma Agrária (Incra), Instituto Nacional do Seguro Social (INSS) e Gmunicipal, sendo a Sesan e o Incra responsáveis pela condução de programas voltados para a área rural - Ater, Fomento, Reforma Agrária, Crédito Fundiário etc. - e o Gmunicipal, a gestão municipal da assistência social responsável pela "entrega" dos serviços socioassistenciais.

O CadÚnico apresenta o maior número de remessa de informações - um total de 26 -, mas não recebe nenhuma informação. Isso significa que o CadÚnico, por ser a base de dados para a seleção de todos os programas, repassa a vários outros atores - gestões de programa, sistemas, entidades fornecedoras de bens/serviços -, mas não recebe de nenhum. Dessa forma, é evidente que, ao não "receber" informações, terá condições limitadas de ser um corretor (broker), dado seu baixo potencial de influenciar, controlar ou dar acesso a outros nós da rede.

O nível restrito de influência do cadastro fica mais evidente ao verificar que este não se encontra entre os atores com mais valores quanto à medida de centralidade de intermediação (betweenness centrality), indicadas na Tabela 4. Essa métrica considera um ator o meio para alcançar outros atores, visto que ele está posicionado nos caminhos geodésicos entre outros pares de atores na rede. Marteleto (2001) afirma que um ator pode ter poucos contatos diretos na rede, mas exercer um importante papel intermediando informações ao controlar o acesso daqueles que circulam na rede e o trajeto que podem percorrer. 


\section{TABELA 4 REDE 2018 (BETWEENESS)}

\begin{tabular}{lcc} 
& Betweenness & nBetweenness \\
\hline Incra & 482,1 & $9 \%$ \\
Sesan & 422,5 & $8 \%$ \\
Sis. Bancário & 373 & $7 \%$ \\
Sitah & 348,3 & $7 \%$ \\
Gmunicipal & 331 & $6 \%$ \\
SIGPBF & 154,5 & $3 \%$ \\
INSS & 142,2 & $3 \%$ \\
Deben & 137,5 & $3 \%$ \\
Prontuário Suas & 137 & $2 \%$ \\
SNAS & 99 & $2 \%$
\end{tabular}

Fonte: Elaborada pelas autoras.

Além dos atores citados acima e que efetivamente têm papel relevante na condução das políticas voltadas à população rural e à assistência social, merecem atenção alguns sub-sistemas, como o Sis. Bancario e o Sitah. O primeiro retrata o sistema bancário que envolve a rede de pagamentos de benefícios (Banco do Brasil, Caixa Econômica Federal e bancos privados); assim, recebe informações de vários programas e devolve dados sobre saques e pagamentos, mas não é um articulador de políticas. De igual forma, o Sitah suporta o Minha Casa, Minha Vida recebendo várias informações de outras bases para verificar a veracidade daquelas prestadas ao CadÚnico. Assim, seu papel é mais de controladoria e/ou auditoria do que efetivamente de transmissão ou articulação de políticas.

Os clusters ou aglomerados são um conjunto de nós fortemente conectados, seja por compartilhar características comuns, seja por estar próximos entre si dentro da rede. As medidas de aglomeração permitem identificar comunidades dentro das redes. Na Tabela 5 foi calculado o coeficiente local que expressa um índice de "grupabilidade" dos nós vizinhos em que o ponto máximo seria a interconectividade com todos os nós. Segundo Recupero, Presutti, Consoli, Gangemi, e Nuzzolese (2015), Watts e Strogatz (1998) introduziram essa métrica como forma de determinar quem forma um mundo pequeno. 
TABELA 5 REDE 2018 (CL3USTER)

$\begin{array}{cc} & \text { nPairs } \\ \text { Cadastro } & 325 \\ \text { Sesan } & 78 \\ \text { Gmunicipal } & 55 \\ \text { Incra } & 55 \\ \text { Sitah } & 36 \\ \text { INSS } & 28 \\ \text { Deben } & 21 \\ \text { Serviço Peti } & 10 \\ \text { Sipra } & 10 \\ \text { Agências INSS } & 10 \\ \text { Prontuário Suas } & 10\end{array}$

Fonte: Elaborada pelas autoras.

Na Figura 3, é perceptível no sociograma a existência de conjuntos de programas que compartilham informações, formando um emaranhado mais denso de nós e linhas, o que poderíamos caracterizar como áreas de maior integração das políticas públicas.

\section{FIGURA 3 SOCIOGRAMA (REDE 2018) - IDENTIFICAÇÃO DE CONJUNTOS}

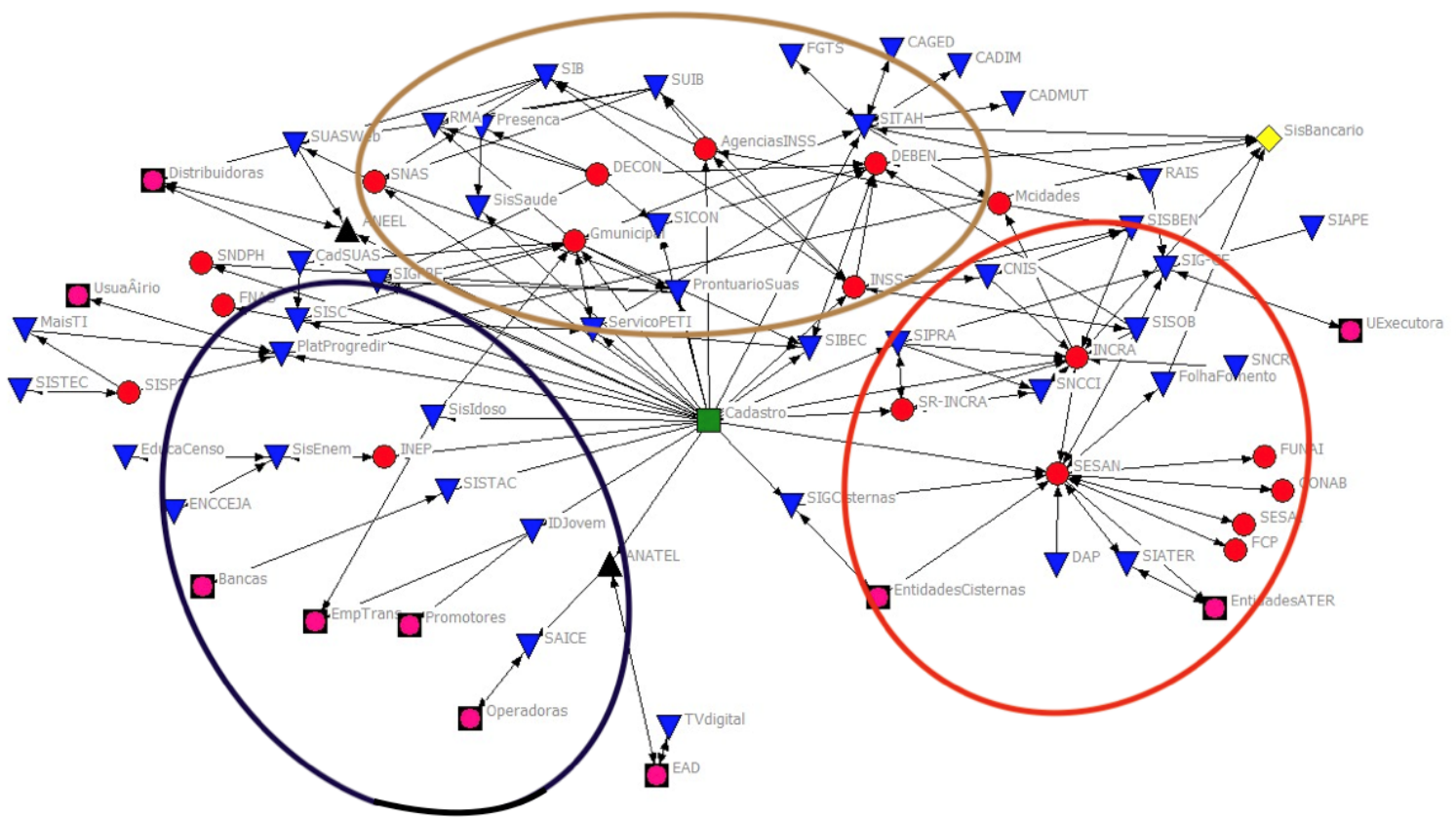

Legenda sociograma de identificação de conjuntos

Gestão municipal - Assistência social

- Políticas rurais

Programas diversos

Fonte: Elaborada pelas autoras. 
O NetDraw permite que se identifique a rede egocentrada de um nó. Percebe-se, nas medidas da Tabela 5, a proeminência que alguns atores adquirem, como os nós Gmunicipal, Sesan e Incra, sendo que Gmunicipal representa as gestões municipais, responsáveis por prestar informações e serviços em especial os socioassistenciais -, Sesan e Incra atuam com políticas sociais voltadas ao meio rural.

A análise do sociogarma da Figura 4 corrobora para evidenciar o papel esperado das prefeituras no pacto federativo brasileiro, como ponto de contato direto com o cidadão, reforçando a ideia de que "os municípios, assim, tornaram-se os gestores fundamentais das políticas sociais no Brasil" (Linhares, Mendes, \& Lassance, 2012); logo, são os "entregadores" dos serviços socioassistenciais. Como esperado, a rede apresenta, além do vínculo com o próprio CadÚnico, ligação com a assistência social por meio de seus sistemas como o Prontuário Suas e o Registro Mensal de Atendimento (RMA) do Suas, que, além dos serviços socioassistenciais, também se conectará ao sistema de solicitação e concessão do BPC (SIB/Suib). O SIGPBF e o Sicon, por sua vez, fornecem à gestão municipal informações para o acompanhamento das famílias em descumprimento das condicionalidades do Programa Bolsa Família. A conexão com o Sitah ocorre em função de parte do processo de seleção das famílias beneficiárias do programa Minha Casa, Minha Vida (faixa 1) ser executado nas gestões municipais da assistência, via módulo existente no próprio CadÚnico.

\section{FIGURA 4 SOCIOGRAMA DA REDE EGOCENTRADA DA GESTÃO MUNICIPAL}

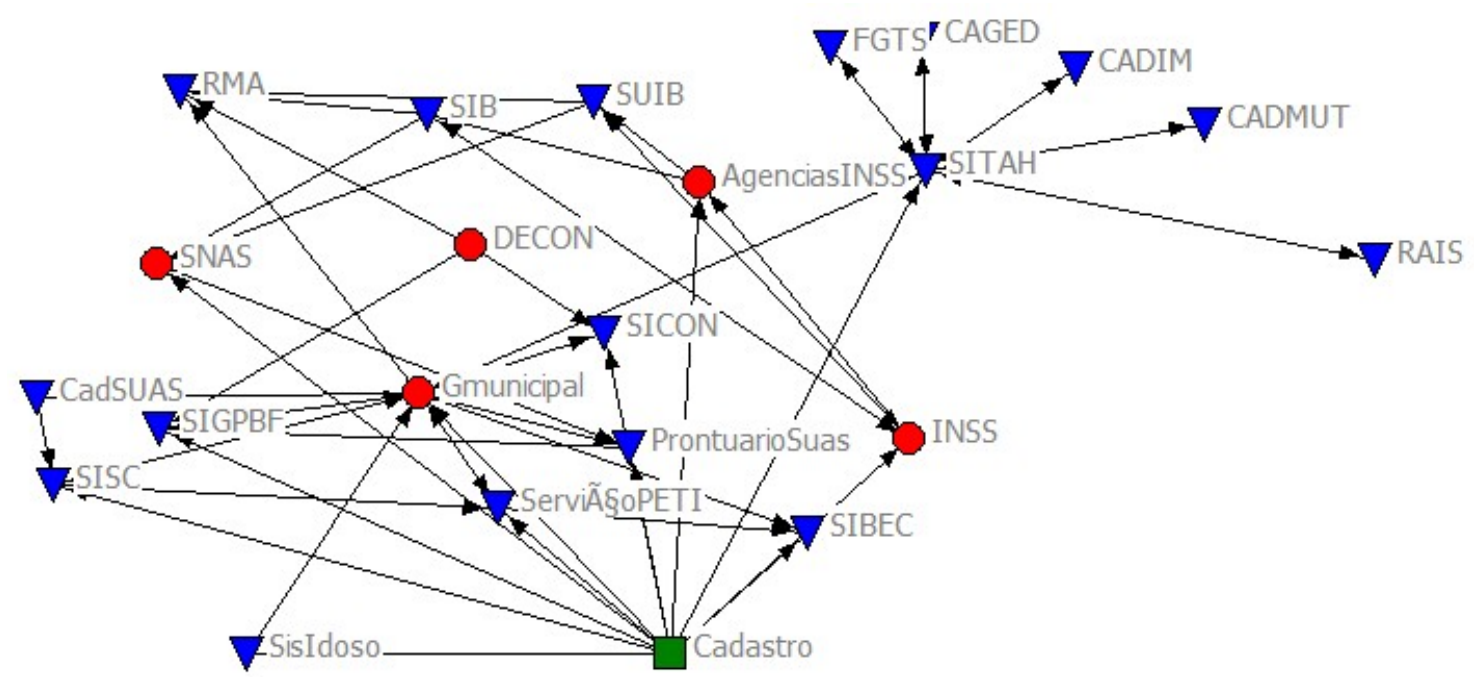

Fonte: Elaborada pelas autoras.

Outro exemplo de maior interação entre nós está no conjunto em que se encontram a Sesan e o Incra, atores que articulam todo o sistema de programas de apoio à área rural, como Cisternas, distribuição de cestas de alimento, Reforma Agrária, Assistência Técnica Rural e Fomento, bem como crédito de instalação para assentados. 


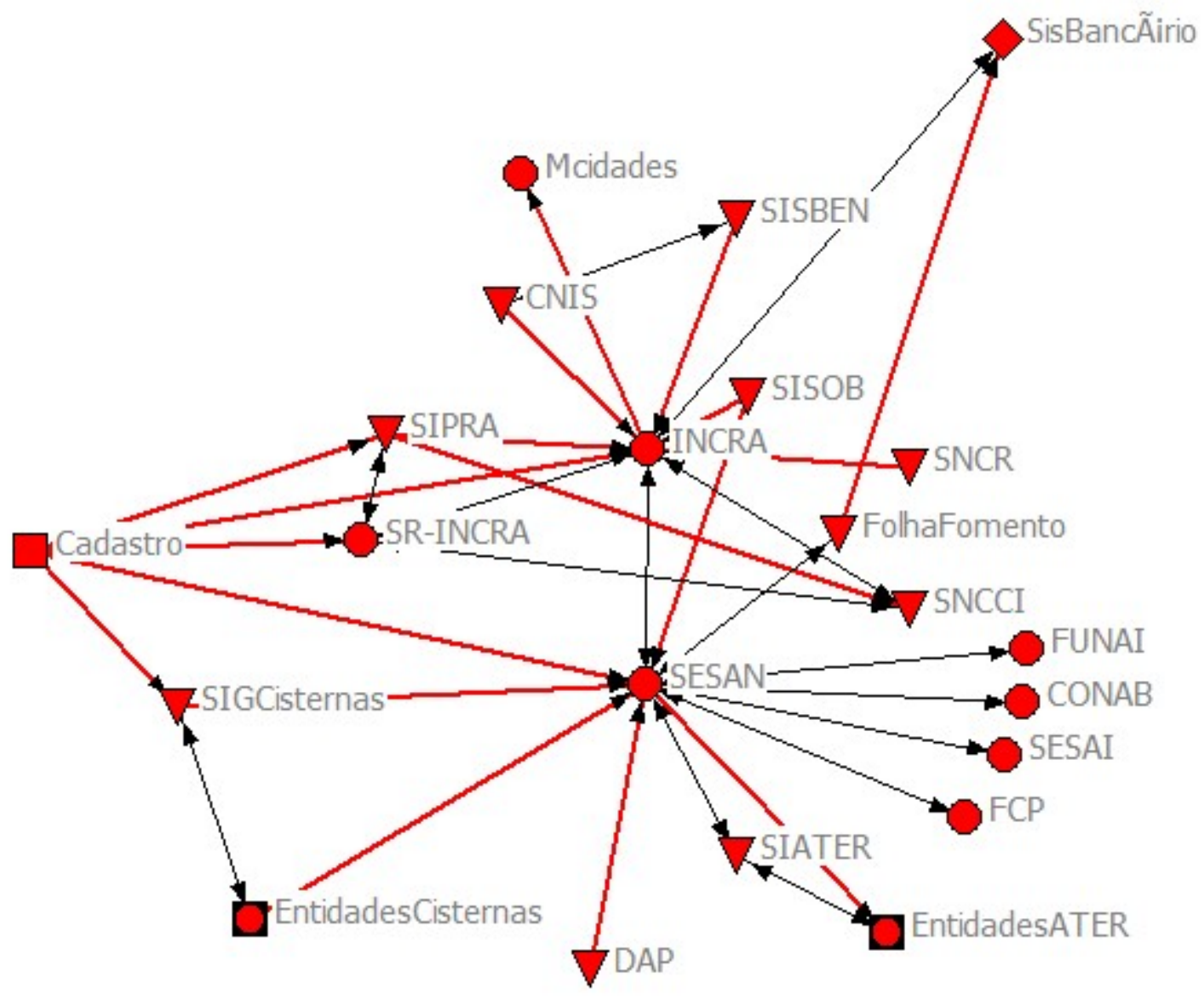

Fonte: Elaborada pelas autoras.

Para fins de comparação, vale observar os dados do sociograma reproduzidos na Figura 6, que representam um conjunto de programas em que há baixa interação entre os atores. O sociograma apresenta linhas que saem do nó do CadÚnico e chegam diretamente às entidades, em sua grande maioria de caráter privado, para o fornecimento de bens ou serviços - é o caso de programas como Tarifa Social de Energia Elétrica, TV Digital, Telefone Popular, Programa Progredir, Identidade Jovem (ID Jovem) etc. Eles representam interações simples e diretas, sem intermediadores, entre o cadastro e os programas provedores de serviços e benefícios sociais que, em geral, são entes privados. 


\section{FIGURA 6 SOCIOGRAMA DA REDE DE PROGRAMAS DIVERSOS}

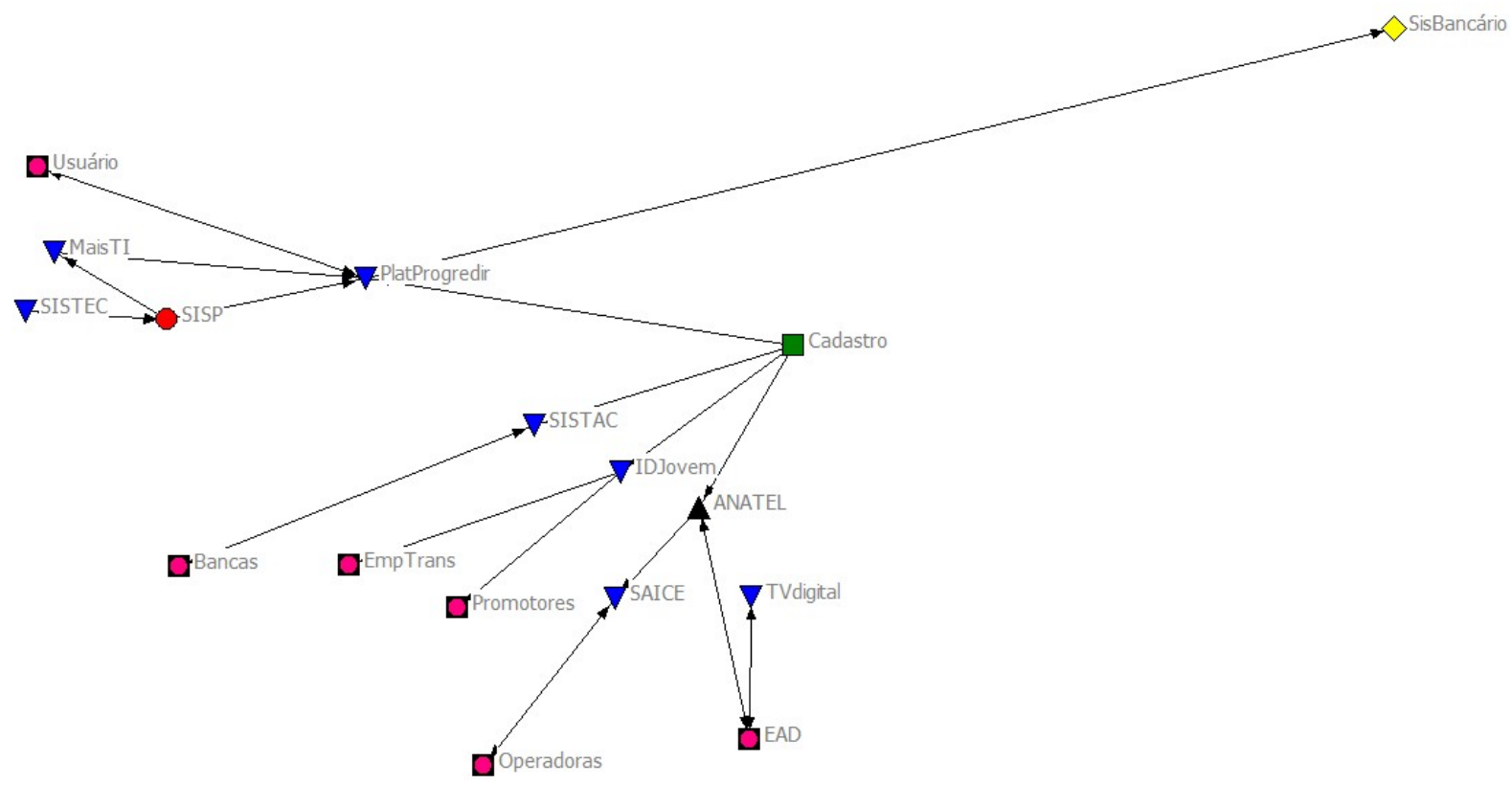

Fonte: Elaborada pelas autoras.

\subsection{Características dos Programas sob a Perspectiva Relacional}

A análise das medidas e dos sociogramas permitiu a identificação de três principais formas relacionais estabelecidas entre os atores da rede, como visto no quadro abaixo.

\section{QUADRO 1 TIPOS DE INTERAÇÃO NA IMPLEMENTAÇÃO}

\begin{tabular}{|c|c|c|}
\hline Grupo A (interação simples) & $\begin{array}{c}\text { Grupo B (sistema de informação/ } \\
\text { intermediários) }\end{array}$ & Grupo C (interações complexas) \\
\hline $\begin{array}{l}\text { Aneel - Tarifa Social de Energia } \\
\text { Elétrica (TSEE) }\end{array}$ & SIB/Suib - BPC & $\begin{array}{l}\text { Incra (Fomento, Ater, PNRA, Crédito de } \\
\text { Instalação) }\end{array}$ \\
\hline Anatel (TV Digital e Telefone Popular) & $\begin{array}{l}\text { Sitah (Sistema Gestor do } \\
\text { Programa Minha Casa, Minha } \\
\text { Vida) }\end{array}$ & $\begin{array}{l}\text { Sesan (Distribuição de Cestas, Ater, Fomento, } \\
\text { Cisternas) }\end{array}$ \\
\hline $\begin{array}{l}\text { Sistac (Isenção de Taxa de Concurso } \\
\text { Público) }\end{array}$ & SIGCisternas & $\begin{array}{l}\text { Decon (condicionalidades do Programa Bolsa } \\
\text { Família) }\end{array}$ \\
\hline ID Jovem (Identidade Jovem) & $\begin{array}{l}\text { PlatProgredir (Programa Progredir } \\
\text { - Microcrédito) }\end{array}$ & $\begin{array}{l}\text { Serviço Peti (Programa de Erradicação do } \\
\text { Trabalho Infantil) }\end{array}$ \\
\hline Sisidoso (Carteira do Idoso) & & $\begin{array}{l}\text { GMunicipal (recebe e repassa do atendimento ao } \\
\text { público serviços e benefícios socioassistenciais) }\end{array}$ \\
\hline
\end{tabular}

Fonte: Elaborado pelas autoras. 
O Grupo A é formado por um conjunto de políticas públicas que têm no CadÚnico sua principal fonte de informação, não mantendo praticamente nenhuma outra interface com outros sistemas/ programas. Assim, são linhas num sentido único que partem do CadÚnico até a borda do sociograma. Não se trata, portanto, de relações integradas, uma vez que a interação entre esses programas se dá apenas com o CadÚnico e em mão única, isto é, o cadastro fornecendo informações aos programas, mas sem a viabilização de integração com outras políticas.

No Grupo B, as informações que saem do cadastro são "carregadas" em outros sistemas agregadores, cujo objetivo é validar as informações do cadastro com dados de outras bases para a seleção de beneficiários. Há um nível de integração promovido por esses sistemas, mas não há formas de interação com outras políticas. Chama a atenção nessa categoria a centralidade dos sistemas informatizados como o Sitah e o SIGCisternas, ambos fornecendo suporte a programas específicos. O Sitah operacionaliza o Programa Minha Casa, Minha Vida e recebe vários dados de diversas fontes, mas apresenta somente uma saída que permite a gestão do programa, funcionando também como "auditoria” dos dados fornecidos ao CadÚnico. Já o SIGCisternas suporta a implantação de cisternas, remetendo informações para a Sesan, que fará a conexão com outras políticas.

Por fim, o Grupo C corresponde ao conjunto de vínculos de políticas com interações mais complexas entre os atores envolvidos e, portanto, um maior nível de integração entre as políticas envolvidas. É o caso das políticas voltadas especialmente à área rural (Sesan e Incra), além da própria gestão municipal e do Sicon/Decon, que interagem não só via sistema, mas também por meio de processos, normas e interações com os gestores de assistência social, saúde e educação, a fim de permitir o acompanhamento das condicionalidades do Programa Bolsa Família.

\section{CONCLUSÃO}

A análise dos instrumentos de implementação é uma forma de "desvendar" a ação pública, uma vez que permite compreender as consequências que tais escolhas acarretaram para o próprio fazer governamental e para os cidadãos. Este estudo buscou explorar a escolha do CadÚnico como instrumento de integração de políticas públicas, seus usos e capacidades de fato para desempenhar tal papel. Do ponto de vista estatal, a análise do contexto relacional do cadastro demonstra diversos desafios para alcançar a finalidade de integração de políticas. Do ponto de vista societal, percebem-se as implicações da baixa integração das políticas para os beneficiários.

As métricas e os sociogramas demonstram que, embora o cadastro seja o principal fornecedor de informações dessa rede, é praticamente nula sua centralidade como receptor de dados de programas e políticas, sugerindo que não há uma reatroalimentação ou integração dos dados que permita ajustes mais precisos sobre a situação das famílias, o que poderia aprimorar a implementação de todas as políticas. De igual forma, como demonstrado nos sociogramas, existe uma distância significativa entre os beneficiários e a gestão da maior parte dos programas e da gestão central do cadastro, o que também inviabilizaria uma melhor implementação dessas políticas.

Os dados levantados pela pesquisa e sintetizados no Quadro 1 apontam a heterogeneidade nos níveis de integração das políticas analisadas. $\mathrm{O}$ cadastro se apresentou como um broker entre a maior parte dos atores da rede. Isto é, tais atores interagem em sua maioria, independentemente da intermediação do CadÚnico, sugerindo que o cadastro não é um instrumento potente de integração 
de políticas públicas, embora haja casos de interações mais complexas em que ele figura como viabilizador de níveis de integração mais elevados entre as políticas públicas.

Dessa forma, o CadÚnico, não obstante tenha sido criado com a finalidade de integrar políticas públicas e seja um importante condicionante para isso, por si só não tem a capacidade de garantir a integração. Percebe-se que as políticas que o utilizam foram concebidas numa lógica de especialização, dificultando a integração. De fato, há pouco ou talvez nenhum reconhecimento dos efeitos simultâneos entre as políticas públicas. Ademais, não há definição de metas e objetivos comuns que poderiam pavimentar o caminho a ser seguido, assim como também não existe monitoramento ou avaliação integrados.

A despeito dos distintos níveis de integração encontrados, há que se reconhecer que, se não existisse o CadÚnico, a possibilidade de integrar políticas seria quase nula. No entanto, outros condicionantes são igualmente relevantes, como arranjos institucionais de coordenação, normas que explicitem os objetivos comuns, capacidades organizacionais que garantam os meios para integração - por exemplo, equipes, equipamentos, sistemas informatizados etc. Devem-se considerar, por exemplo, as implicações em não ter uma identificação única do cidadão, o que dificulta sobremaneira o cruzamento de dados e a identificação do acesso às várias políticas. Em outras palavras, sem a identificação efetiva do cidadão e de sua família, bem como as informações socioeconômicas que o CadÚnico propicia, dificilmente se poderá integrar de fato um conjunto de políticas que atinjam as diferentes formas de vulnerabilidade.

Tais evidências levam à conclusão de que há potencialidade de integração na rede do CadÚnico. Contudo, a interação hoje observada entre as políticas é heterogênea, com casos de praticamente nenhuma relação com outros atores e casos de alta interação em campos específicos.

Futuras pesquisas sugeridas incluem a exploração desses casos para compreensão dos demais condicionantes, a fim de uma maior integração entre políticas, assim como a continuidade da análise longitudinal, visando identificar outros fatores determinantes para a integração, como as mudanças político-institucionais que fazem nascer ou morrer as políticas ou dão maior ou menor centralidade a elas.

Por fim, sugerem-se pesquisas que explorem as limitações do próprio cadastro, uma vez que, já que uma maior permeabilidade de sua gestão às demandas dos programas e, especialmente, dos próprios beneficiários pode imprimir grandes transformações não apenas de eficiência, mas de efetividade desses programas para a melhoria da vida da população mais vulnerável do Brasil. 


\section{REFERÊNCIAS}

Barca, V., \& Chirchir, R. (2014). Single registries and integrated MISs: De-mystifying data and information management concepts. Camberra, Australia: Department of Foreign Affairs and Trade.

Bruijn, J. A., \& Hufen, H. A. M. (1998). The traditional approach to policy instruments. In B. G. Peters, \& F. K. M. V. Nispen. (Eds.), Public policy instruments: Evaluating the tools of public administration. New York, NY: Edward Elgar.

Direito, D., Koga, N., Lício, E., \& Chaves, J. (2016, julho). O Cadastro Único como instrumento de articulação de políticas sociais (Working paper número 145). Brasília, DF: Centro Internacional de Políticas para o Crescimento Inclusivo. Recuperado de https://ipcig.org/pub/port/OP327PT_O_Cadastro_ Unico_como_instrumento_de_articulacao_de_ politicas_sociais.pdf

Direito, D., Koga, N., Lício, E., \& Chaves, J. (2018). As possibilidades e os limites do Cadastro Único como mecanismo de coordenação federativa e de intersetorialidade de políticas públicas. In D. Ximenes. (Org.), Implementação de políticas públicas: questões sistêmicas, federativas e intersetoriais. Brasília, DF: Enap.

Dobuzinskis, L., \& Howlett, M. (Eds.) (2018). Policy analysis in Canada. Bristol, UK: Policy Press.

Emirbayer, M. (1997). Manifesto for a Relational Sociology. The American Journal of Sociology, 103(2), 281-317. Recuperado de https://www.jstor.org/ stable/10.1086/231209

Freeman, L. (2002). The development of social network analysis: a study in sociology of science. North Charleston, SC: Booksurge.

Furtado, B. A., Sakowski, P. A. M., \& Tóvolli, M. (2015). Modelagem de sistemas complexos para políticas públicas. Brasília, DF: Ipea.

Hatala, J. P. (2006). Social network analysis in human resource development: a new methodology. Human Resource Development Review, 5(1), 45-71.

Hood, C. (1983). The tools of Government. London, UK: Macmillan.

Hood, C. (2007). Intellectual obsolescence and intellectual makeovers: Reflections on the tools of government after two decades. Governance, 20(1), 127-144.
Howlett, M. (2000). Managing the "hollow state": Procedural policy instruments and modern governance. Canadian Public Administration, 43(4), 412-431.

Howlett, M. (2009). Governance modes, policy regimes and operational plans: A multi-level nested model of policy instrument choice and policy design. Policy Sciences, 42(1), 73-89.

King, D. (2007). The American State and Social Engineering Policy Instruments in Affirmative Action. Governance, 20, 109-126.

Knoke, D., \& Yang, S. (2008). Social network analysis. London, UK: Sage Publications.

Lascoumes, P., \& Le Galès, P. (2005). Gouverner par les instruments. Paris, France: Presses de Sciences Po.

Lascoumes, P., \& Le Galès, P. (2007). Introduction: understanding public policy through its instruments-from the nature of instruments to the sociology of public policy instrumentation. Governance, 20, 1-21.

Linder, S., \& Peter, G. (1990). The Design of Instruments for Public Policy. In N. Westport. (Ed.), Policy Theory and Policy Evaluation. Westport, CO: Greenwood Press.

Linhares, P. T. F., Mendes, C. C., \& Lassance, A. (2012). Federalismo à brasileira: questões para discussão. Brasília, DF: Ipea.

Marques, E. C. (2006). Redes sociais e poder no estado brasileiro: aprendizado a partir das políticas urbanas. Revista Brasileira de Ciências Sociais, 21(60), 15-41.

Marteleto, R. (2001). Análise de redes sociais: aplicação nos estudos de transferência da informação. Ciência da Informação, 30(1), 71-81.

Peters, B. G. (2015). Pursuing horizontal management: The politics of public sector coordination. Kansas, KS: University Press of Kansas.

Peters, B. G., \& Nispen, F. K. M. van (eds). (1998). Public policy instrumentes: Evaluating the tools of public administration. Nova York, NY: Edward Elgar.

Recupero, D. R., Presutti, V., Consoli, S., Gangemi, A., \& Nuzzolese, A. G. (2015). Sentilo: frame-based sentiment analysis. Cognitive Computation, 7(2), 211-225. 
Sabatier, P. A. (1986). Top-Down and Bottom-up approaches to implementation research: a critical analysis and suggested synthesis. Journal of Public Policy, 6(1), 21- 48.

Salamon, L. (2002). The Tools of Government: A Guide to the New Governance. New York, NY: Oxford University Press.

Scott, J. (1992). Social network analysis. Newbury Park, CA: Sage Publications.

Silva, A., Matheus, R., Parreiras, F., \& Parreiras, T. (2006). Análise de redes sociais como metodologia de apoio para a discussão de interdisciplinaridade na ciência da informação. Recuperado de www.scielo. br/pdf/ci/v35n1/v35n1a09
Tomaél, M. I., \& Marteleto, R. M. (2006). Redes sociais: posições dos atores no fluxo da informação. Encontros Bibli - Revista Eletrônica de Biblioteconomia e Ciência da Informação, 11(1), 75-91.

Watts, D. J., \& Strogatz, S. H. (1998). Collective dynamics of 'small-world'networks. Nature, 393(6684), 440-442.

Wasserman, S., \& Faust, K. (1994). Social network analysis: Methods and applications. Cambridge, UK: Cambridge University Press.

Wellman, B. (1997). Structural analysis: From method and metaphor to theory and substance. Contemporary Studies in Sociology, 15, 19-61.

\section{Denise do Carmo Direito}

https://orcid.org/0000-0002-2323-2846

Doutoranda em Ciência Política na Universidade de Brasília (UnB), área de concentração Política e Instituições; Mestre em Ciência Política pela Universidade de Brasília (UnB), área de concentração Democracia e Sociedade; Membro da carreira de Especialista em Políticas Públicas e Gestão Governamental, desde 2002.

E-mail: denisedireito.dd@gmail.com

\section{Natalia Massaco Koga}

iD

https://orcid.org/0000-0003-0143-8373

Doutora em Ciência Política pela University of Westminster, Reino Unido; Pesquisadora da Diretoria de Estudos e Políticas do Estado, das Instituições e da Democracia (Diest) do Instituto de Pesquisa Econômica e Aplicada (IPEA); É membra da carreira de Especialista em Políticas Públicas e Gestão Governamental; Professora do Mestrado Profissional em Governança e Desenvolvimento da Escola Nacional de Administração Pública (ENAP). E-mail: natmkoga@gmail.com 